PALEO

Revue d'archéologie préhistorique

$22 \mid 2011$

Varia

\title{
Crache perforée dans le Gravettien du sire (Mirefleurs, Puy-de-Dôme)
}

Étude archéozoologique, technologique et fonctionnelle

Perforated Gravettian red deer canine from Sire (Mirefleurs, Puy-de-Dôme):

Archaezooological, technological and use-wear analysis

Francesco d'Errico et Solange Rigaud

\section{OpenEdition}

Journals

Édition électronique

URL : http://journals.openedition.org/paleo/2172

DOI : $10.4000 /$ paleo.2172

ISSN : 2101-0420

Éditeur

SAMRA

Édition imprimée

Date de publication : 1 décembre 2011

Pagination : 301-310

ISSN : 1145-3370

Référence électronique

Francesco d'Errico et Solange Rigaud, «Crache perforée dans le Gravettien du sire (Mirefleurs, Puy-deDôme) », PALEO [En ligne], 22 | 2011, mis en ligne le 19 avril 2012, consulté le 07 juillet 2020. URL : http://journals.openedition.org/paleo/2172 ; DOI : https://doi.org/10.4000/paleo.2172

\section{(c) (i) (9)}

PALEO est mis à disposition selon les termes de la licence Creative Commons Attribution - Pas d'Utilisation Commerciale - Pas de Modification 4.0 International. 


\title{
CRACHE PERFORÉE DANS LE GRAVETTIEN DU SIRE (Mirefleurs, Puy-de-Dôme) : étude archéozoologique, technologique et fonctionnelle
}

\author{
Francesco D’ERRICO ${ }^{(1,2)}$, Solange RIGAUD ${ }^{(1)}$
}

\begin{abstract}
Résumé : Le gisement gravettien ancien du Sire a livré une unique crache de cerf utilisée comme objet de parure. L'analyse archéozoologique, technologique et tracéologique de cette pièce révèle qu'il s'agit de la canine droite d'un cerf male âgé d'environ dix ans. La couronne a été modifiée par abrasion et la racine perforée par raclage et par rotation, avant d'être partiellement sciée à la hauteur de la perforation et fracturée par flexion. L'usure de la perforation témoigne du port de l'objet étalé dans le temps. Des traces de pigments s'observent sur l'ensemble de l'objet, y compris sur les surfaces de fracture. L'extrême rareté du Cerf dans la faune du gisement et le fait que la pièce a été conservée après le sciage indiquent que les Gravettiens attribuaient une valeur certaine à cette crache. Une revue bibliographique des parures gravettiennes rapproche Le Sire des sites localisés dans la zone méridionale de l'aire de distribution de ce technocomplexe.

Mots-clés : cerf, canine, parure, symbolisme, Paléolithique supérieur.

Abstract: Perforated Gravettian red deer canine from Sire (Mirefleurs, Puy-de-Dôme): Archaezooological, technological and use-wear analysis. The Early Gravettian site of Sire, Mirefleurs, Puy-de-Dôme, dated to 27.3 ka 14C BP, has yielded a unique red deer canine, used as personal ornament. Zooarchaeological and morphological analysis indicate that the tooth comes from a ten years old stag. Use-wear analysis indicates that the crown of the tooth was deliberately abraded, probably to remove the enamel. The root was scraped with a lithic tool before being pierced by rotation. Use-wear on the perforation suggests the object was used as element of a beadwork (necklace, bracelet...) or sewn onto a garment or similar item. The root was subsequently notched and snapped. Use wear on the break indicates the object was curated after removal of the root, perhaps in a skin bag, and may have been used again as ornament with a different type of attachment. Microscopic residues of red pigment are present across the entire surface of the tooth. This complex history of use and the rarity of red deer remains in the site's faunal assemblage, which is dominated by horse remains, support the view that the object was highly valued by its Gravettian makers. A survey of the literature on red deer canine used as ornaments during the Gravettian identifies similarities with other southern European sites.
\end{abstract}

Key-words: red deer, canine, personal ornaments, symbolism, Upper Paleolithic.

\section{Introduction}

Le gisement du Sire a été découvert en 1969 au cours de travaux agricoles par Pierre Daniel (Surmely et al. 2003). Le gisement a fait l'objet de plusieurs sondages de 2000 à 2002, puis a été fouillé à partir de 2003. Les fouilles ont été menées avec les méthodes classiques d'enregistrement des données spatiales et le sédiment a été tamisé à l'eau avec des tamis à mailles fines (Surmely et al. 2008, 2011).
La prédominance des microgravettes, la présence discrète des pièces esquillées et des pointes de Font-Robert ont conduit les fouilleurs à attribuer le gisement à une phase très ancienne du Gravettien (Hays et Surmely 2005 ; Surmely et al. 2008). Le croisement des données typotechnologiques et zooarchéologiques suggère que le gisement a vraisemblablement fonctionné comme halte de chasse ou campement résidentiel à proximité de sites d'abattages de chevaux (Surmely et al. 2008).

(1) UMR 5199 PACEA, Institut de Préhistoire et de Géologie du Quaternaire, Université Bordeaux I, Avenue des Facultés, F-33405

Talence, France

(2) Institute of Archaeology, History, Cultural Studies and Religion, University of Bergen, Norway 
Une crache de cerf perforée, qui fait l'objet de notre étude, constitue l'unique objet de parure retrouvé sur le gisement. La canine de cerf a été récoltée lors de la campagne de fouille 2005 et provient du niveau supérieur, couche 3a, secteur $\mathrm{B}$, daté à $27.330 \pm 264 \mathrm{BP}(\mathrm{Wk}-17110)$ (Surmely et al. 2003, 2008, 2011). Malgré son caractère unique, cet objet nous renseigne sur les préférences des chasseurscueilleurs qui ont occupé le gisement, les techniques utilisées pour la confection d'objets d'ornementation ainsi que leur port. Cette dent percée peut ainsi nous éclairer sur le monde social et symbolique de la population concernée, sur son territoire et sur ses possibles réseaux d'échange.

Les craches de Cerf se prêtent à une série d'analyses spécialement développées pour l'étude de cette catégorie de matériel (d'Errico et Vanhaeren 2002). En s'appuyant sur un référentiel actuel, il est ainsi possible d'identifier l'âge et le sexe des cerfs sur lesquels les canines ont été prélevées. Couplée à une analyse technologique et microscopique des perforations, cette méthode a déjà montré son potentiel informatif et permis de proposer des hypothèses sur l'organisation sociale des sociétés de la fin du Paléolithique supérieur et du Mésolithique (d'Errico et Vanhaeren 1999, 2000 ; Vanhaeren 2002 ; Vanhaeren et d'Errico 2001, 2003a, 2003b, 2005 ; Rigaud et al. 2010).

Dans cette étude, nous croiserons ces méthodes pour reconstituer les différentes étapes de la vie sociale de cet objet de parure, depuis son prélèvement sur l'animal jusqu'à sa perte. II s'agira également de vérifier en quelle mesure l'utilisation de ce type de parure par les hommes de Le Sire s'intègre dans le monde culturel gravettien et si sa présence dans ce site peut contribuer à identifier des tendances régionales au sein de ce technocomplexe.

\section{Méthodologie}

\section{Analyse morphométrique}

Le protocole suivi se base sur l'enregistrement de variables morphométriques et morphologiques discriminantes pour l'attribution de l'âge et du sexe des cerfs sur lesquels les dents ont été prélevées (d'Errico et Vanhaeren 2002). Ces critères ont été établis par l'analyse de référentiels de comparaison constitués de canines prélevées sur des animaux d'âge et de sexe connus, provenant de l'île de Rum (Clutton-Brock et al. 1982, 1997 ; Benton et al. 1995 ; Coulson et al. 1997 ; Kruuk et al. 1999 ; d'Errico et Vanhaeren 2002) et de la réserve royale de Richmond (d'Errico et Vanhaeren 2002). Cinq variables morphologiques et neuf variables métriques ont été enregistrées sur chacune des canines (fig. 2). Les variables morphologiques (fig. 1) comprennent cinq stades d'usure occlusale, trois stades de développement de la racine, l'état de fermeture de la cavité pulpaire et l'enlèvement par l'usure du lobe disto-linguo-cervical (proéminence localisée sur l'extrémité postérieure de la face linguale de la couronne).

Les variables métriques comprennent la largeur, la longueur et l'épaisseur de la couronne, la largeur et la longueur de la facette d'usure occlusale ainsi que la largeur maximale, la largeur à l'apex, l'épaisseur et la longueur des racines (fig. 1). Le diamètre de la perforation a également été relevé.
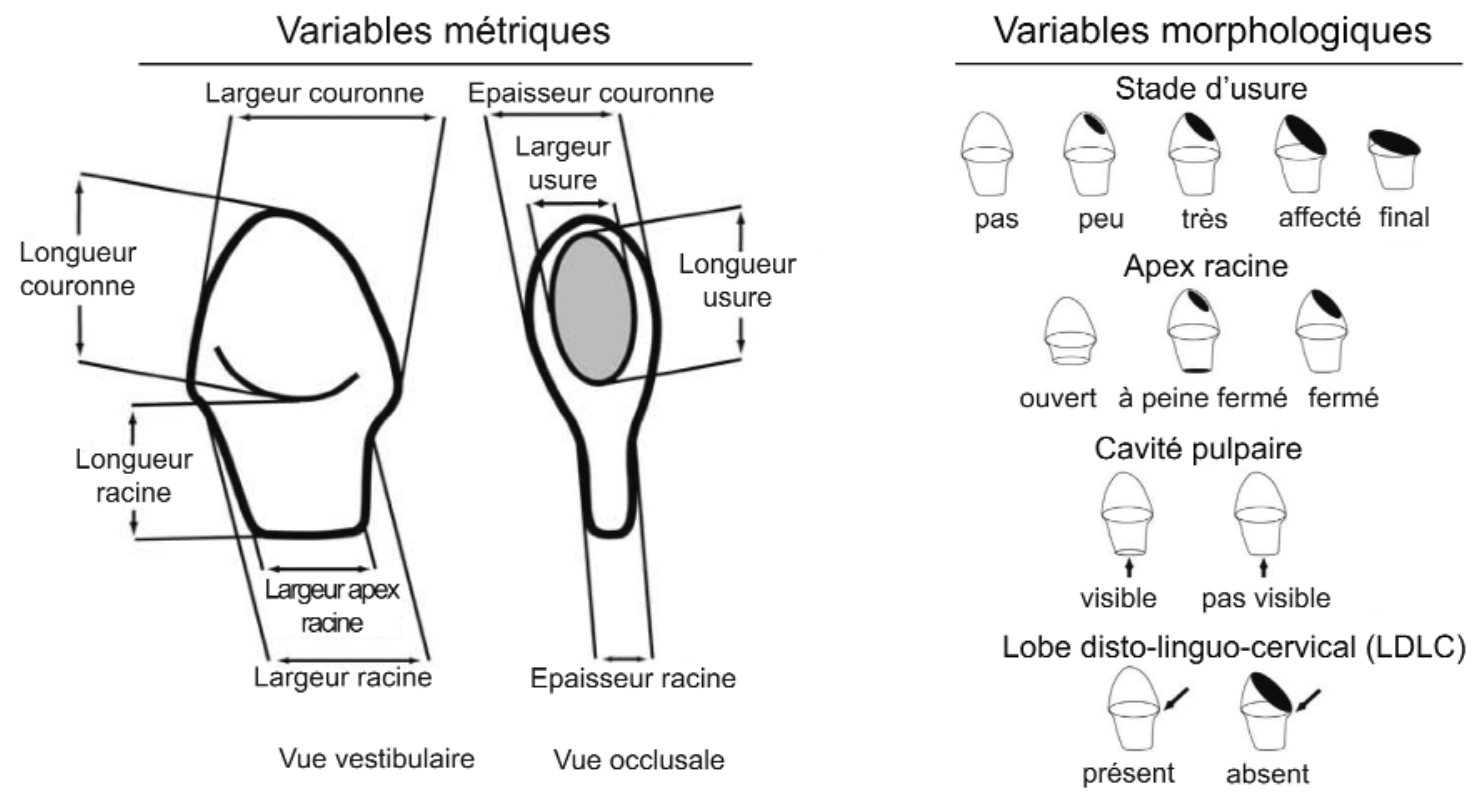

Figure 1 - Variables métriques et morphologiques enregistrées sur les canines (modifié d'après d'Errico et Vanhaeren 2002).

Figure 1 - Metric and morphological variables recorded on red deer canines (modified after d'Errico and Vanhaeren 2002). 


\section{Analyse technologique}

Un microscope Leica Z6 APO motorisé, équipé d'une camera DFC420 et d'un système d'acquisition et de traitement d'image LAS, a été utilisé pour documenter les modifications d'origine naturelle et anthropique observées sur la dent. En combinant des images prises à différentes hauteurs ce microscope permet d'obtenir des photos entièrement au point. Les modifications observées sur les faces linguales et vestibulaires ont été comparées afin d'évaluer la variabilité gestuelle de l'artisan. La localisation et le degré de développement des usures dues au port des objets ont été enregistrés ainsi que la présence de résidus de pigment.

\section{Résultats}

\section{Identification taxinomique}

De par sa morphologie, la dent est identifiée comme une canine supérieure droite de Cervus elaphus (fig. 2).

\section{État de conservation}

La racine est presque entièrement absente (fig. 2). La surface de la pièce est altérée et présente des cupules de dissolutions témoignant d'une attaque acide. On observe également des encroûtements sur la couronne et sur la racine au niveau de la perforation.

\section{Identification du sexe}

Sur la base de critères morphologiques, la crache a été attribuée au sexe mâle (fig. 2).

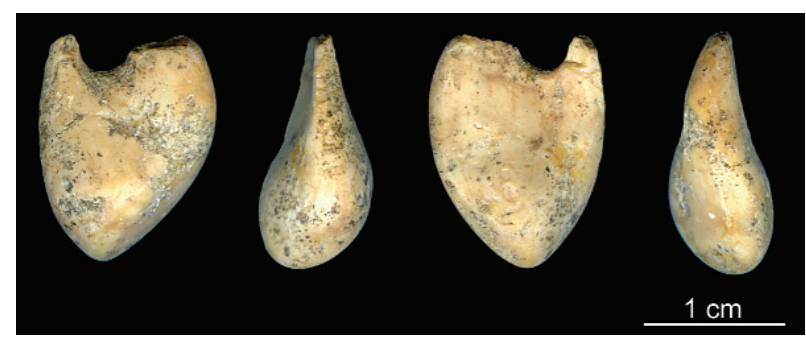

Figure 2 - Photos des faces linguale, distale, vestibulaire et mésiale de la canine supérieure droite de cerf récoltée sur le gisement du Sire.

Figure 2 - Photos of the lingual, distal, vestibular and mesial aspects of the red deer canine from Sire.
Cette attribution est confirmée par les dimensions du diamètre vestibulo-lingual et mésio-distal de la racine qui s'intègrent parfaitement au sein de la variabilité rencontrée chez les mâles des deux populations de référence (tabl. 1, fig. 3).

\section{Estimation de l'âge}

Deux méthodes ont été mises au point pour le calcul de l'âge des cerfs à partir de leurs craches. La première utilise une équation prenant en compte des variables métriques. La deuxième se base sur les stades d'usure de la couronne et le croisement de cette information avec des données liées au développement de la dent. La première méthode ne peut pas être appliquée à la dent de Le Sire car l'absence de la racine empêche la mesure de sa longueur, variable nécessaire pour l'application de l'équation. La méthode des stades d'usure, quant à elle, range cette dent au sein du stade 4, correspondant à un âge compris entre 9 et 13 ans. En considérant l'état d'avancement de l'usure pour ce stade, le fait que la cavité pulpaire de la racine ne soit pas visible, et que le lobe disto-linguo-cervical soit encore préservé, implique que l'âge de l'animal soit plus proche de la limite inférieure de cette tranche, soit entre 9 et 11 ans (fig. 4).

\section{Aménagement de la couronne}

Les faces vestibulaire et linguale de la couronne sont traversées par des stries parallèles, orientées perpendiculairement à l'axe de la dent. Ces stries semblent avoir été produites en frottant la couronne sur une matrice abrasive homogène à grains fins (fig. $5 \mathrm{~g}, \mathrm{~h}$ ). Cette technique a pu servir à éliminer l'émail, de manière à donner à la surface de la dent un aspect régulier. Ces stries sont recoupées par d'autres stries plus récentes, orientées de façon aléatoire, résultant vraisemblablement du port de l'objet et de l'action de particules du sédiment. Ce recouvrement atteste de l'ancienneté des marques d'abrasion.

\section{Perforation}

L'usure résultant du port de l'objet et l'action des agents post-dépositionnels ont contribué à effacer la plupart des traces laissées au cours de cette phase d'aménagement de l'objet. Néanmoins, en se basant sur les traces encore visibles, on peut établir que la perforation a suivi deux étapes. Les faces vestibulaire et linguale de la racine ont tout d'abord été raclées à l'aide d'un outil lithique, en suivant un mouvement parallèle ou légèrement oblique à

\begin{tabular}{|c|c|c|c|c|c|c|c|c|c|c|c|c|c|}
\hline & & & & \multirow{2}{*}{\multicolumn{5}{|c|}{ Couronne }} & \multirow{2}{*}{\multicolumn{4}{|c|}{ Racine }} & \multirow[b]{2}{*}{ Perforation } \\
\hline \multirow[b]{2}{*}{ Site } & \multirow[b]{2}{*}{ Année } & \multirow[b]{2}{*}{ Secteur } & \multirow[b]{2}{*}{ Couche } & & & & & & & & & & \\
\hline & & & & Long. & larg. & Ep. & L. usure & I. us ure & Long. & larg. & Ep. & I. apex & Diam. max. pert Diam. min. perf \\
\hline Le Sire & 2005 & B & $3 a$ & 11,4 & 11,54 & 6,56 & 9,57 & 7,23 & na & 10,02 & 3,52 & na & 3,05 \\
\hline
\end{tabular}

Tableau1 - Variables métriques relevées sur la canine du Sire (en mm).

Table 1 - Morphometric data recorded on the canine from Sire. 


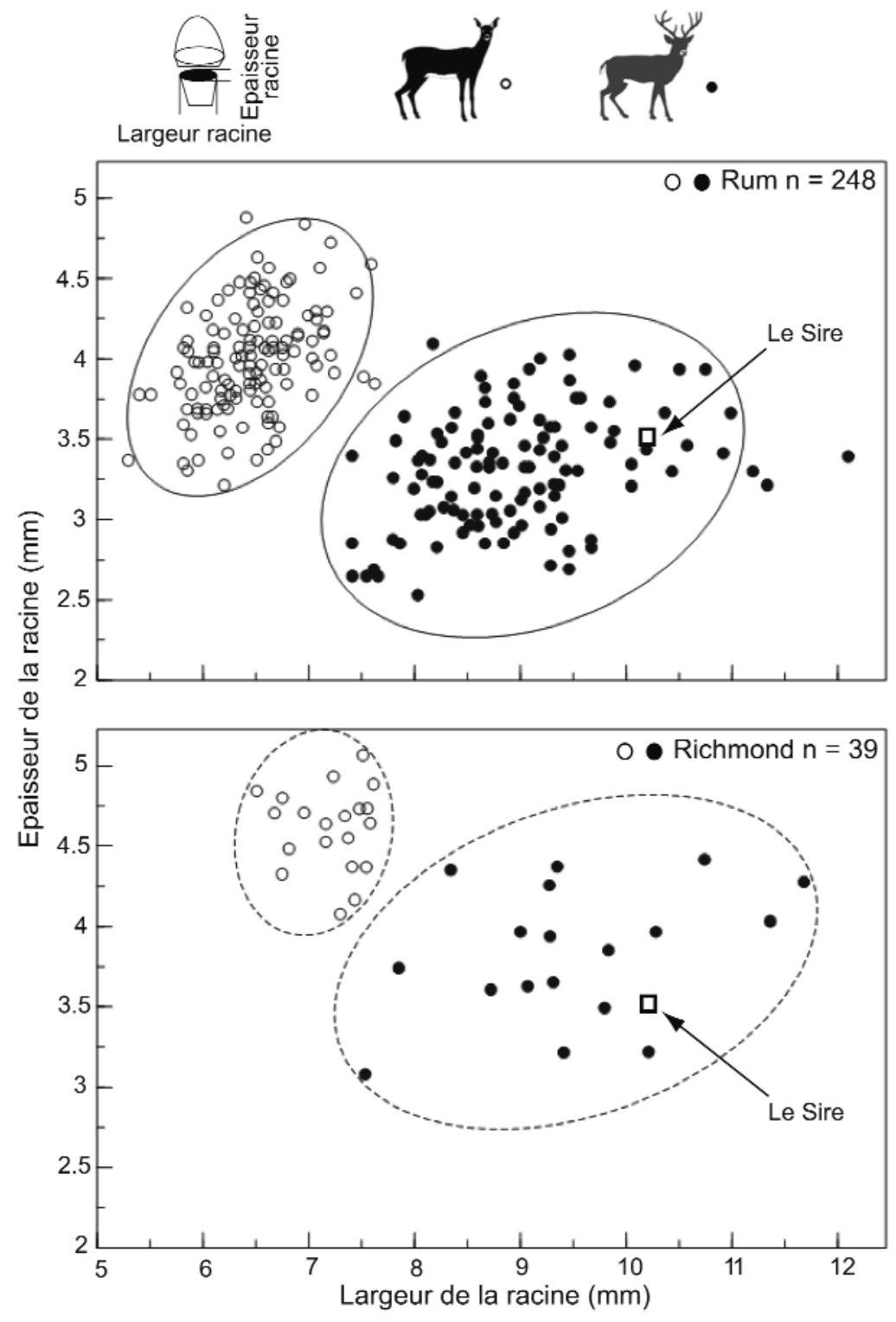

Figure 3 - Projection de la canine supérieure du Sire dans les nuages de points qui mettent en relation la largeur et l'épaisseur de la racine des mâles et des femelles de deux populations actuelles de cerfs de sexe connu. Ellipse de confiance $=95 \%$.

Figure 3 - Root width and thickness on male and female red deer canines from two comparative collections and the Sire tooth. Confidence Ellipse $=95 \%$. l'axe de la dent (fig. 5a, b, c). Ce raclage a eu pour objectif d'amincir sur les deux faces les zones à perforer et de créer une concavité permettant de stabiliser la pointe au cours de la phase suivante. Celle-ci a alors consisté à perforer la racine par rotation en approfondissant et élargissant le creux, probablement en alternance sur les deux faces (fig. $5 d$ ). II est difficile d'établir, à cause du caractère unique de l'objet, si la localisation désaxée de la perforation, proche du bord mésial de la racine (fig. 2), est accidentelle ou le résultat d'une volonté de l'artisan.

\section{Suspension}

Une partie du pourtour de la perforation présente des encroûtements empêchant l'identification de traces d'utilisation sur l'ensemble de la surface (fig. 5d).
Cependant, les zones libres de concrétions sont lustrées, témoignant de l'usure de la perforation lors de la suspension. L'atténuation des traces de raclage en se rapprochant de la perforation et la discrétion des traces de rotation suggèrent également que la crache a été portée (fig. 5a, b, c).

\section{Sciage}

Des traces de sciage, visibles exclusivement à l'échelle microscopique, sont présentes de part et d'autre de la perforation sur la face linguale de la racine (fig. 5e, f). Elles sont orientées perpendiculairement à l'axe d'allongement de la dent. L'encoche produite par sciage ne traverse pas entièrement la racine, ce qui indique que l'artisan s'est limité à produire une encoche avant de fracturer la dent par flexion. 


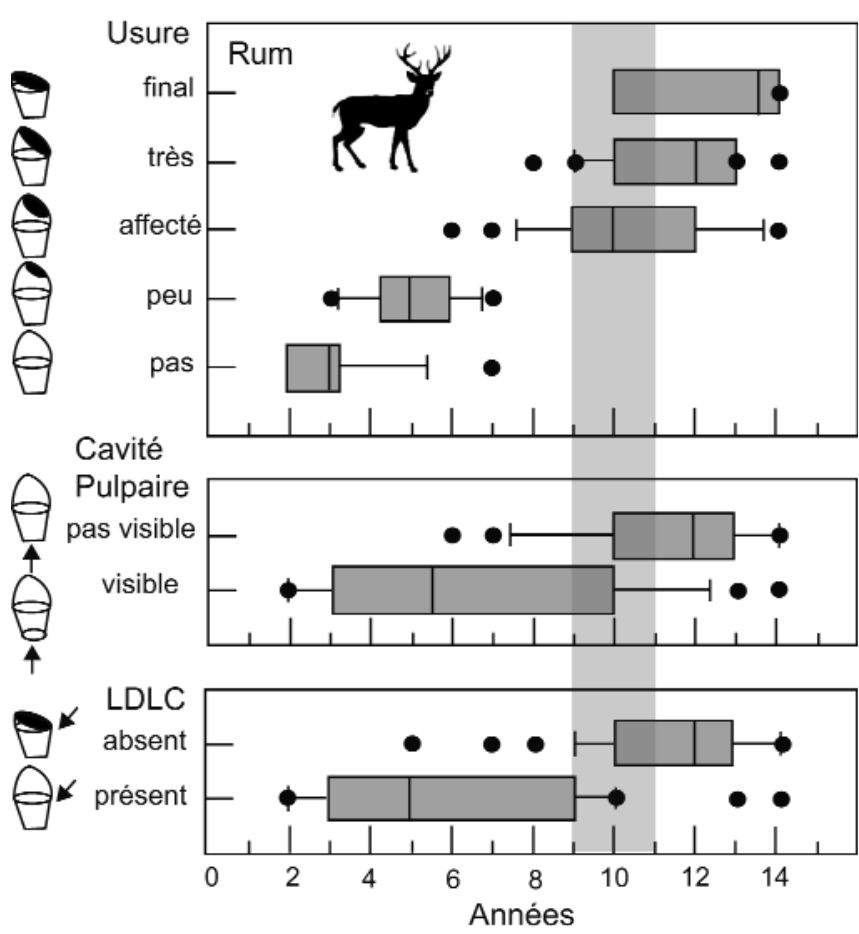

Figure 4- Evolution des variables morphologiques en fonction de l'âge des cerfs (modifié d'après d'Errico et Vanhaeren 2002). La bande grisée correspond à la plage de recouvrement des variables relevées sur la canine du Sire.

Figure 4 - Changes during life of reed deer canine morphological features (modified after d'Errico and Vanhaeren 2002). The area in grey identifies the combination of features observed on the Sire specimen.

\section{Transport}

Les zones sciées et les surfaces de fracture par flexion présentent un poli intense indiquant que la fracture a été régularisée par abrasion et que la pièce a été conservée et peut être réutilisée après l'ablation de la racine (fig. 6a, $b, c, d)$.

\section{Pigment}

Des traces microscopiques de pigment rouge ont été détectées autour de la perforation, sur les traces de sciage et sur les surfaces de fracture adjacentes (fig. 5c, d).

\section{Discussion}

Les résultats obtenus permettent de restituer les comportements impliqués dans la confection et l'utilisation de la crache de Cerf du Sire. Ces éléments contribuent à reconstituer les étapes de la vie sociale de l'objet.

L'analyse morphométrique et l'analyse des stades d'usure de la couronne montrent que la canine a été prélevée sur un cerf mâle âgé entre 9 et 11 ans. Aucune trace d'extraction n'a été observée sur la dent. Celle-ci a pu être collectée sur la carcasse d'un animal mort naturellement, ou extraite du maxillaire d'un individu fraîchement chassé. La deuxième hypothèse est la plus probable car ces dents ont tendance à se détacher rapidement de la mâchoire en cas de mort naturelle.

La faune du niveau supérieur du gisement auquel appartient la couche $3 a$ ayant livré la crache est largement dominée par le Cheval (Surmely et al. 2008). Un très faible nombre de restes a été attribué au Cerf. La signification environnementale de chacune de ces deux espèces est communément présentées comme sensiblement différente: le Cheval serait caractéristique d'un milieu ouvert alors que le Cerf signalerait un environnement forestier ou partiellement boisé (Delpech et al. 1983 ; Griggo 1995). Cependant, Cheval et Cerf sont reconnus comme des ongulés qui, au Pléistocène, possédaient la plus grande valence écologique, c'est-à-dire qui présentaient les plus larges tolérances climatiques (Delpech 1990, 1999). Dans ce contexte, ces deux espèces ont pu survivre, ensembles, sous des conditions sévères et en des lieux particuliers comme les contreforts de zones montagneuses par exemple (Delpech 1990). Or, le spectre faunique de la couche $3 a$ indique qu'à l'époque de l'occupation du site le milieu était ouvert, de type steppe froide (Delpech et al. 1983). Aussi, si l'environnement était alors plus propice au Cheval qu'au Cerf, tous deux auraient néanmoins pu se maintenir dans ce milieu très contraignant alors que d'autres ongulés, moins tolérants, se seraient grandement raréfiés. C'est donc sans doute sur un individu ayant vécu dans leur environnement proche que les Gravettiens du Sire ont prélevé la crache qu'ils ont ensuite perforée.

L'analyse technologique montre que la couronne a été modifiée par abrasion, probablement avant la perforation de la racine, réalisée par raclage et rotation avec une pointe lithique. La pièce a ensuite été portée, suspendue ou cousue sur un vêtement. Le port a été suffisamment long pour développer un poli capable d'estomper les traces de perforation et d'abrasion de l'émail. Les quelques exemples expérimentaux de reproduction des usures dues au port (d'Errico 1993) suggèrent que cette période a duré de quelques mois à plusieurs années. 

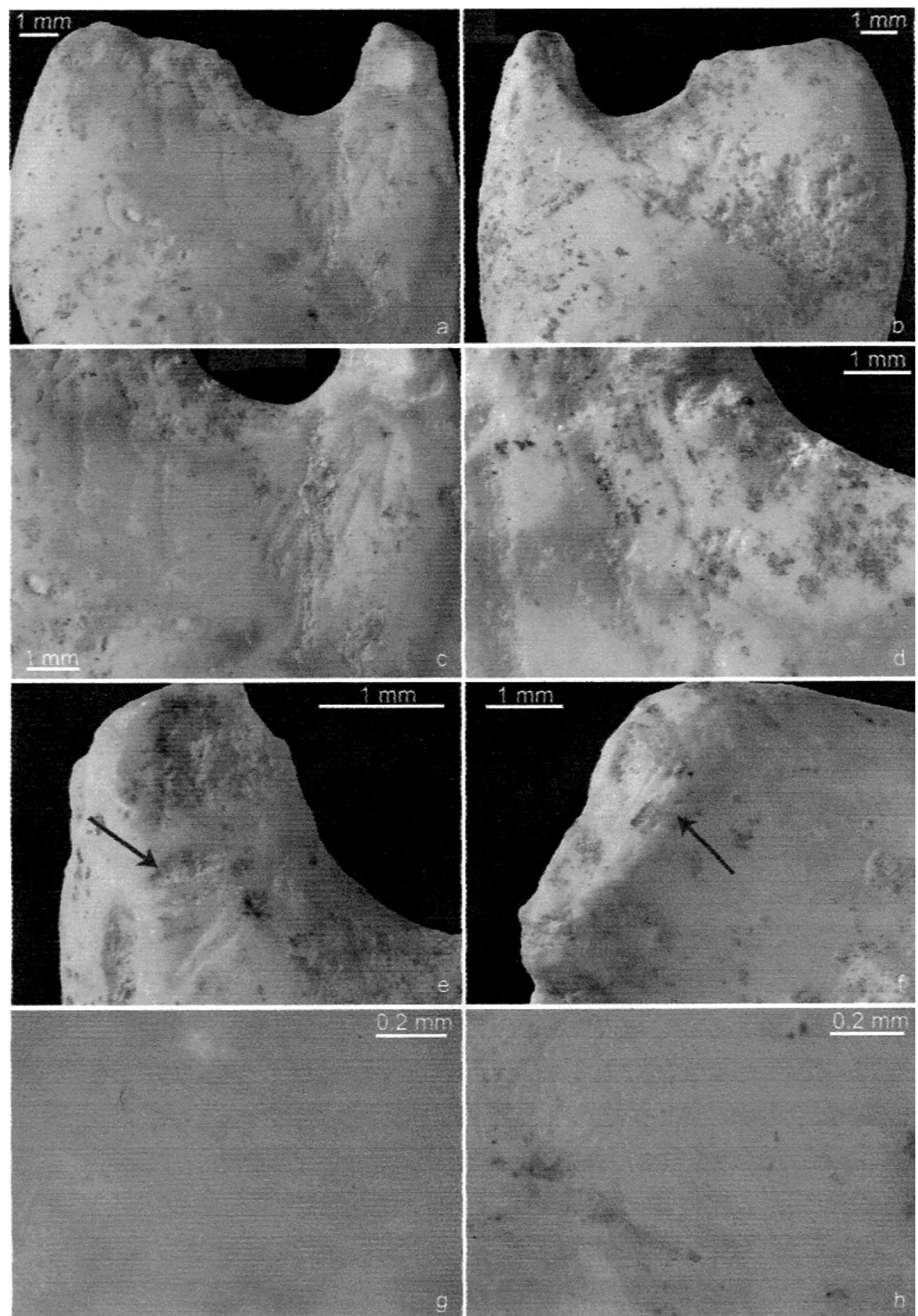

Figure 5 - Traces d'aménagements sur la couronne et la racine de la crache. $a-b$ : face vestibulaire et linguale de la dent; $c$ : traces de raclage sur la face vestibulaire ; $d$ : trace de rotation sur la face vestibulaire ; e : trace de sciage sur le bord disto-lingual de la racine (flèche) ; $f$ : traces de sciages sur le bord mésio-lingual de la racine (flèche) ; $g$ : traces d'abrasion sur la face vestibulaire de la couronne; $h$ : traces d'abrasion sur la face linguale de la couronne.

Figure 5 - Anthropogenic modifications identified on the root and the crown of the canine. a-b: vestibular and lingual aspects of the tooth; c: scraping on the vestibular aspect of the root; d: rotation marks on the vestibular aspect of the root; e: sawing marks on the disto-lingual aspect of the root (arrow); $f$ : sawing marks on the disto-lingual aspect of the root (arrow); g: abrasion marks on the vestibular aspect of the crown; $h$ : abrasion marks on the lingual aspect of the crown. 

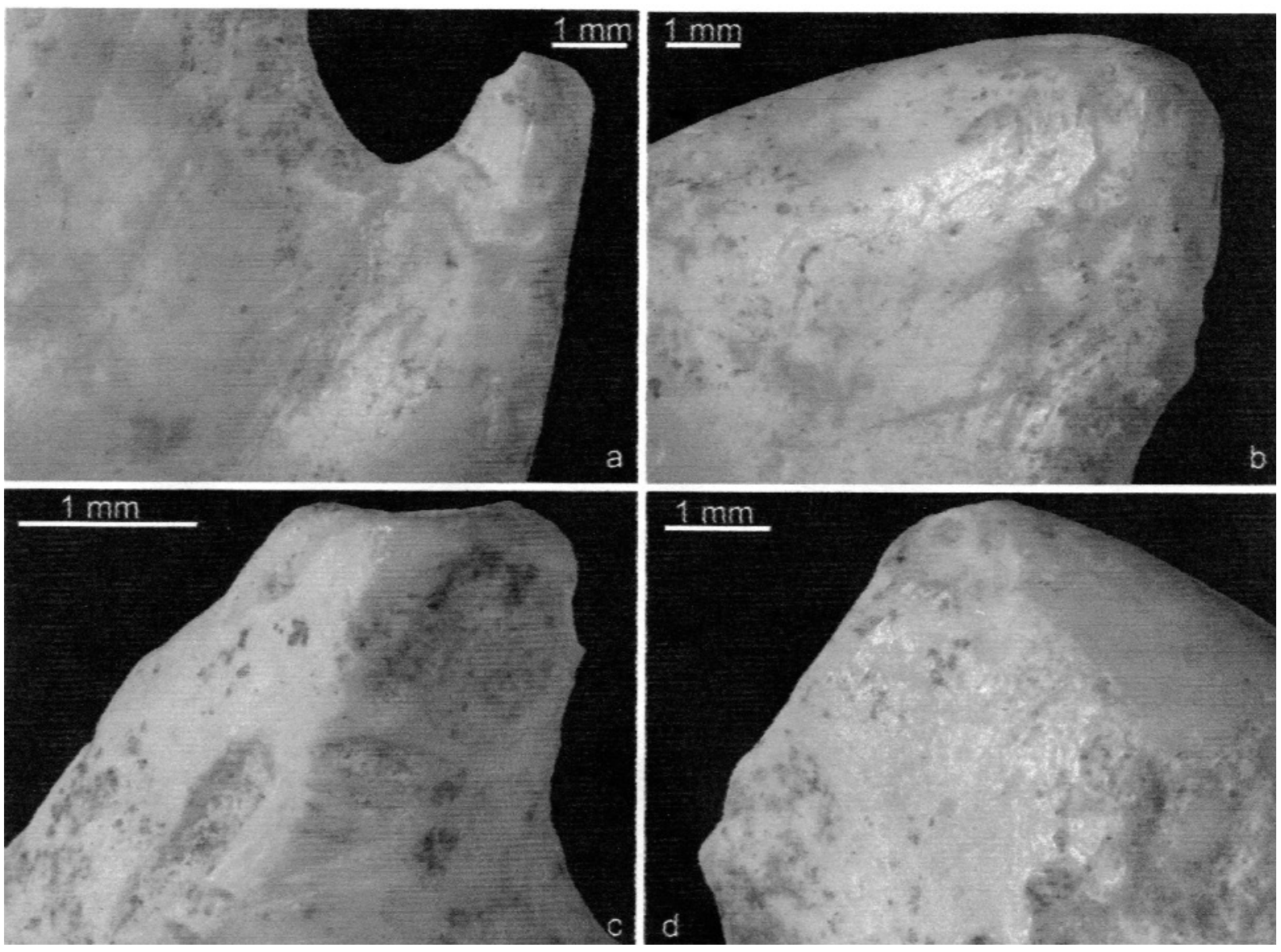

Figure 6 - Polis présents sur les bords distal $(a, c)$ et mésial $(b, d)$ de la racine.

Figure 6 - Use-wear on the distal $(a, c)$ and mesial $(b, d)$ aspects of the root.

Dans un second temps, la racine de la dent a été enlevée par sciage et flexion. Le poli observé sur les fractures suggère qu'après avoir été sciées les fractures ont été régularisées par polissage et la crache a été conservée, probablement dans un contenant en peau, en vue d'une utilisation ultérieure comme objet de parure, ou en tant qu'objet répondant à une autre fonction (amulette, talisman...). Elle a pu être perdue à ce stade, ou intégrée à un nouveau système de parure avant d'être perdue. Dans le dernier cas, le nouveau système de parure auquel la crache était destinée devait être conçu de façon à fixer l'objet sans avoir besoin d'une perforation pour l'attache. Cela a pu être réalisé grâce à un mastic ou à un fil serré. Les traces de pigment présentes sur la racine pourraient remonter à la première phase d'utilisation de l'objet, à son transport après sciage ou à son possible recyclage dans une deuxième parure.

La pièce de Le Sire n'est pas le premier cas d'une crache de cerf sciée découverte en contexte gravettien. Une des quatre craches associées à la sépulture de l'enfant de
Lagar Velho (Portugal), indirectement datée aux environs de 24500 ans BP, est également sciée (Vanhaeren et d'Errico 2003). Elle diffère néanmoins de celle du Sire pour l'âge et le sexe de l'individu dont elle provient, une biche d'âge avancé, et le fait que le sciage, proche de l'apex, fait le tour de la racine en dégageant une gorge qui a vraisemblablement été utilisée pour la suspension de l'objet. Synthèse des éléments de parure recensés dans les sépultures gravettiennes montre que les craches sont présentes dans 20 sépultures réparties entre l'Italie et le Portugal (Vanhaeren et d'Errico 2003). En revanche, elles sont absentes des sépultures localisées au nord de cette zone. Ce constat donne un parfum méridional à ce type d'objet de parure pendant le Gravettien. Néanmoins, des craches ont été également découvertes dans des sites gravettiens allemands, français, italiens, espagnols, portugais et moldaves (Taborin 2000). L'absence d'une base de données des parures gravettiennes rend difficile pour l'instant d'explorer le rôle de ces objets dans la définition d'ensemble régionaux comme cela a été fait récemment pour l'Aurignacien (Vanhaeren et d'Errico 2006). 


\section{Conclusion}

Nos résultats indiquent que la crache de cerf perdue sur le site du Sire a eu une longue vie sociale. L'attention avec laquelle elle a été modifiée, perforée, portée, réaménagée et ensuite conservée pour être peut être réutilisée, suggère que cette pièce devait représenter un objet de valeur pour son ou ses possesseurs. La présence de cet objet de parure dans des nombreux sites et sépultures contemporains atteste des liens symboliques que les habitants du Sire partageaient avec les autres Gravettiens et en particulier ceux du sud de l'Europe.

\section{Remerciements}

Nous remercions Frédéric Surmely pour nous avoir confié l'étude de l'objet. Cette recherche a été financée par le European Research Council (FP7/2007/2013/ERC Grant TRACSYMBOLS $n^{\circ} 249587$ ) dans le cadre du programme FP7.

\section{Références bibliographiques}

BENTON TG., GRANT A., CLUTTON-BROCK TH. 1995 Does environmental stochasticity matter? Analysis of red deer life-histories on Rhum Evolutionary Ecology, 9, p. 559-74.

CLUTTON-BROCK TH., GUINNESS FE., ALBON SD. 1982 Red Deer. Behavior and Ecology of Two Sexes. Chicago: The University of Chicago Press. 378 p.

CLUTTON-BROCK TH., ROSE KE., GUINNESS FE. 1997 Density-related changes in sexual selection in red deer. Proceedings of the Royal Society of London, 264, p. 1509-16.

COULSON T., ALBON SD., GUINNESS FE., PEMBERTON J., CLUTTON-BROCK TH. 1997 - Population substructure, local density and calf winter survival in red deer (Cervus elaphus). Ecology, 78, p. 852-63.

DELPECH F., DONARD E., GILBERT A., GUADELLI J.-L., LE GALL O., MARTINI-JACQUIN A., PAQUEREAU M.-M., PRAT F., TOURNEPICHE J.-F. 1983 - Contribution à la lecture des paléoclimamts quaternaires d'après les données de la Paléontologie en milieu continental. Bulletin de l'Institut de Géologie du Bassin d'Aquitaine, 34 et CNRS, Cahiers du Quaternaire, nº spécial, p. 165-177.

DELPECH F. 1990 - The range distributions of some ungulate mammals during the Dryas I (Recent Würm) in Aquitaine. Paleoecological implications. Geobios, 23 (2), p. $221-230$

DELPECH F. 1999 - Biomasse d'ongulés au Paléolithique et inférences sur la démographie. Paléo, 11, p. 19-42.

D'ERRICO F. 1993 - La Vie Sociale de l'Art Mobilier Paléolithique. Manipulation, Transport, Suspension des Objets en Os, Bois de Cervidés, Ivoire. Oxford Journal of Archaeology, p. 145-174.
D'ERRICO F., VANHAEREN M. 1999 - Les méthodes d'analyse de l'art mobilier paléolithique : quelques exemples issus de la région cantabrique. Anthropologie et Préhistoire, p. 31-45.

D'ERRICO F., VANHAEREN M. 2000 - Mes morts et les morts de mes voisins. Le mobilier funéraire de l'Aven des Iboussières et l'identification de marqueurs culturels à l'Epipaléolithique. Epipaléolithique et Mésolithique : Les derniers chasseurs-cueilleurs d'Europe occidentale. Actes du colloque international de Besançon (octobre 1998). Presses Universitaires Franc-Comtoises: Besançon, p. 325-342.

D'ERRICO F., VANHAEREN M. 2002 - «Criteria for Identifying Red Deer (Cervus elaphus) Age and Sex from Their Canines. Application to the Study of Upper Palaeolithic and Mesolithic Ornaments. Journal of Archaeological Science, , p. 211-239.

GRIGGO C. 1995 - Significations paléoenvironnementales des communautés animales pléistocènes reconnues dans l'abri Suard (Charente) et la grotte de Bois-Ragot (Vienne) : Essai de quantification de variables climatiques. Bordeaux: Université de Bordeaux $1220 \mathrm{p}$.

HAYS M. et SURMELY F. 2005 - Réflexions sur la fonction des microgravettes et la question de l'utilisation de l'arc au Gravettien ancien. Paleo, 17, p. 145-155.

KRUUK LEB., CLUTTON-BROCK TH., ALBON SD., PEMBERTON JM., GUINNESS FE. 1999 - Population density affects sex ratio variation in red deer. Nature, 399, p. 459-61.

RIGAUD S., D'ERRICO F., VANHAEREN M. 2010 - LoS objetos de adorno personal asociados al esqueleto mesolítico Braña-2. In Md León (Ed.), Los hombres mesolíticos de la Cueva de la Braña-Arintero (Valdeugueros, León). Leon: Junta de Castilla y León, p. 62-81.

SURMELY F., ALIX P., COSTAMAGNO S., DANIEL P., MURAT R. et al. 2003 - Découverte d'un gisement du Gravettien ancien au lieu-dit le Sire (Mirefleurs, Puy-deDôme). Bulletin de la Société préhistorique française, 100, p. 29-39.

SURMELY F., COSTAMAGNO S., HAYS M., ALIX P. 2008 Le Gravettien et le Protomagdalénien en Auvergne. Paleo, 20, p. 73-98.

SURMELY F., BALLUT C. 2011 - Le site gravettien ancien du Sire (Mirefleurs, Puy-de-Dôme). Les données lithiques, chronologiques et sédimentaires. In : N. Goutas, L. Klaric, D. Pesesse, P. Guillermin (Eds), A la recherche des identités gravettiennes. Actualités, questionnements et perspectives. Actes de la table ronde sur le gravettien en France et dans les pays limitrophes, Aix-en-Provence (6-8 octobre 2008). Paris, SPF, p. 311-28, (Mémoire de la Société Préhistorique Française $n^{\circ}$ LII). 
TABORIN Y. 2000 - Gravettian body ornaments in Western and Central Europe. In : W. Roebroeks, M. Mussi, J. Svoboda, K. Fennema (Eds), Hunter of the Golden Age, University of Leiden, Leiden p. 135-141.

VANHAEREN M. 2002 - Les fonctions de la parure au Paléolithique supérieur : de l'individu à l'unité culturelle. Bordeaux :Université de Bordeaux 1, $355 \mathrm{p}$.

VANHAEREN M., D'ERRICO F. 2001 - La parure de l'enfant de la Madeleine (fouilles Peyrony). Un nouveau regard sur l'enfance au Paléolithique supérieur. Paleo, p. 201-237.

VANHAEREN M., D'ERRICO F. 2003 - Le mobilier funéraire de la Dame de Saint-Germain-la-rivière et l'origine paléolithique des inégalités. Paleo, 15, p. 195-238.
VANHAEREN M., D'ERRICO F. 2003 - The Body Ornaments Associated with the Burial. Portrait of the Artist as a Child. J. Zilhao et E. Trinkaus (Eds.), The Gravettian human skeleton from the Abrigo do Lagar Velho and its archaeological context. Lisbonne, Instituto Portugues de Archeologia, . 154-186 (de Arqueologia ${ }^{\circ} 22$ ).

VANHAEREN M., D'ERRICO F. 2005 - Grave goods from the Saint-Germain-la-Rivière burial: Evidence for social inequality in the Upper Palaeolithic. Journal of Anthropological Archaeology, p. 117-134. 